\title{
Diphtheria Toxoid/Tetanus Toxoid Vaccine Adsorbed
}

National Cancer Institute

\section{Source}

National Cancer Institute. Diphtheria Toxoid/Tetanus Toxoid Vaccine Adsorbed. NCI

Thesaurus. Code C91718.

A vaccine containing detoxified tetanus toxoid and detoxified diphtheria toxoid adsorbed on aluminum phosphate with active immunizing activity against diphtheria and tetanus. Intramuscular injection with this vaccine activates the immune system to develop antibodies against tetanus toxin and diphtheria toxin. 